\title{
IgE sialylation: unravelling a key anaphylactic mediator
}

\author{
Beatriz Moya ${ }^{1}$, Chiara Tontini ${ }^{2}$, and Alexandra Santos ${ }^{3}$ \\ ${ }^{1}$ Hospital Universitario 12 de Octubre \\ ${ }^{2}$ Azienda Ospedaliero Universitaria Ospedali Riuniti Umberto I G M Lancisi G Salesi \\ ${ }^{3}$ King's College London
}

October 23, 2020

\section{Groundbreaking Discoveries in Immunology}

Title : IgE sialylation: unravelling a key anaphylactic mediator

Authors : Beatriz Moya ${ }^{\mathrm{a}}$, Chiara Tontini ${ }^{\mathrm{b}}$ and Alexandra Santos ${ }^{\mathrm{c}, \mathrm{d}}$, e, f

a. Allergy Service. Hospital Universitario 12 de Octubre, Madrid, Spain

b. Lydia Becker Institute for Immunology and Inflammation, Faculty of Biology, Medicine and Health, University of Manchester, Manchester, UK

c. Department of Women and Children's Health (Paediatric Allergy), School of Life Course Sciences, Faculty of Life Sciences and Medicine, King's College London, London, UK

d. Peter Gorer Department of Immunobiology, School of Immunology and Microbial Sciences, King's College London, London, UK

e. Children's Allergy Service, Guy's and St Thomas' Hospital, London, UK

f. Asthma UK Centre of Allergic Mechanisms of Asthma, London, UK

Correspondence to : Beatriz Moya. Allergy Service. Hospital Universitario 12 de Octubre, Madrid, Spain

Email: drbeatrizmoya@gmail.com

Abbreviations: Ig, Immunoglobulin; Fab, antigen-binding fragments; Fc, fragment crystallizable region; FceRI, Fc epsilon receptor I; MC, mast cells.

Word count: $637 / 1000$

\section{Main text}

Immunoglobulin (Ig) E antibodies are heterotetrameric glycoproteins. They are formed by two antigenbinding fragments (Fab) and a fragment crystallizable region (Fc) (1) with seven N-linked glycosylation sites distributed across the heavy chains. $(2,3)$ Allergen cross-linking of IgE bound to high affinity IgEreceptors (i.e. , FceRI) on mast cells (MCs) and basophils induces the release of mediators that cause allergic symptoms.(4) However, it is largely unknown why patients present a broad spectrum of clinical manifestations and, paradoxically, why many individuals produce allergen-specific IgE without developing allergic symptoms. This indicates that relevant information is still missing about IgE functionality.

Glycosylation is required for proper antibody maturation and determines the specific biological properties of each antibody class (e.g., antigen affinity, Fc receptor binding, downstream immunological activity).(1) Data in cancer and autoimmunity show that glycosylation affects the interaction between Igs and its receptors, thus modulating the pro- or anti-inflammatory properties of Igs in disease-specific patterns.(1) Significantly less is 
known about the role of IgE glycosylation in allergy.(1) Niki et al . found that Galectin-9, a lectin expressed by several MCs, suppressed effector cell degranulation by binding to IgE glycans.(5) Furthermore, Shade et al.identified a single glycan in the $\operatorname{IgE} \mathrm{C} 3$ domain that was essential for triggering anaphylaxis in mice and LAD2 cells (a MC line that derived from a mastocytosis patient).(6) However, the precise mechanisms of this interaction were not fully understood. Recent data by Shade et al . provide novel insights on how $\operatorname{IgE}$ glycosylation determines disease-specific allergic responses.(7)

Shade et al . recently studied the IgE glycosylation pattern of peanut-allergic individuals and non-atopic donors (Figure 1). Human LAD2 MCs were sensitized with serum IgE from these two cohorts and activated by anti-IgE crosslinking. MCs sensitized with IgE from non-atopic individuals degranulated less as compared to MCs sensitized with IgE from peanut-allergic patients. Using glycopeptide mass spectrometry, they determined that certain IgE glycosylation sites (N140- and N265-linked complex glycans terminating in galactose) were enriched in IgE from non-atopic subjects while terminal sialic acids were enriched at the N168 and N265 IgE glycosylation sites in peanut-allergic subjects. By analysing the glycan content of IgE from non-atopic and peanut-allergic subjects, they demonstrated that the galactose and sialic acid contents of $\operatorname{IgE}$ constituted strong predictors of allergic disease.(7)

In addition to studying glycosylation patterns of total IgE, Shadeet al. sought to explore the effects of sialic acid removal from IgE. They observed an attenuation of effector cell degranulation usingin vivo and in vitro models (Figure 1). Interestingly, they showed that allergen bound to asialylated IgE did not activate MCs. These results suggest that the removal of sialic acid from IgE may expose an inhibitory glycan that reduces signalling downstream the FceRI receptor. Using in vivo models, they assessed the therapeutic potential of modulating sialic acid content. By fusing a neuraminidase enzyme towards the $\mathrm{N}$ terminus of IgE Fc Ce2-4 domains to remove sialic acid from IgE-bearing cells, they showed an attenuation of allergeninduced anaphylaxis. (7) (Figure 1) These findings provide evidence for the possibility for new therapeutic strategies targeting IgE sialylation to regulate acute allergic responses. Moreover, the role of glycosylation in allergic reactions appears to extend beyond IgE. A recent study by Petry et al. assessed the impact of IgG glycosylation in anaphylaxis. Their data suggests that enriched blood IgG Fc N-sialylation may regulate the expression of the inhibitory receptor FcrRIIB and protect from IgG-mediated and IgG-FcrRIIB-controlledIgE-mediated allergic reactions.(8)

In summary, Shade et al . provide new compelling insights into the glycobiology of IgE and its effect on allergic responses. Modification of IgE glycosylation patterns could potentially lead to a reduction in the severity of allergic reactions, including anaphylaxis. Sialylation and desialylation may well present a promising diagnostic and treatment strategy for peanut allergy and other IgE-mediated allergic diseases but also to empower the protective inflammatory function of IgE in other pathologies.(9)

Figure 1. Increased terminal sialylation is detected on allergic $\operatorname{IgE}$ and galactose on non-atopic $\operatorname{IgE}$. Compared to sialylated-human-IgE-sensitized MCs, asialylated-human-IgE-sensitized MCs showed a reduced degranulation following allergen challenge. Sensitization to asialylated-mouse-IgE produced a reduced temperature loss following a challenge. In vitro, sensitized MCs with OVA-specific sialylated-human-IgE were incubated with NEUFce, or an IgE isotype control, and challenged with OVA. NEUFce reduced OVA-induced degranulation. In vivo, NEUFce treatment of mice sensitized with asialylated-mouse-IgE showed a reduced temperature drop after a challenge, compared to those with sialylated-mouse-IgE. Ig, Immunoglobulin; MC, mast cells; NEUFce, neuraminidase enzyme towards the $\mathrm{N}$ terminus of IgE Fc; OVA, ovalbumin. 


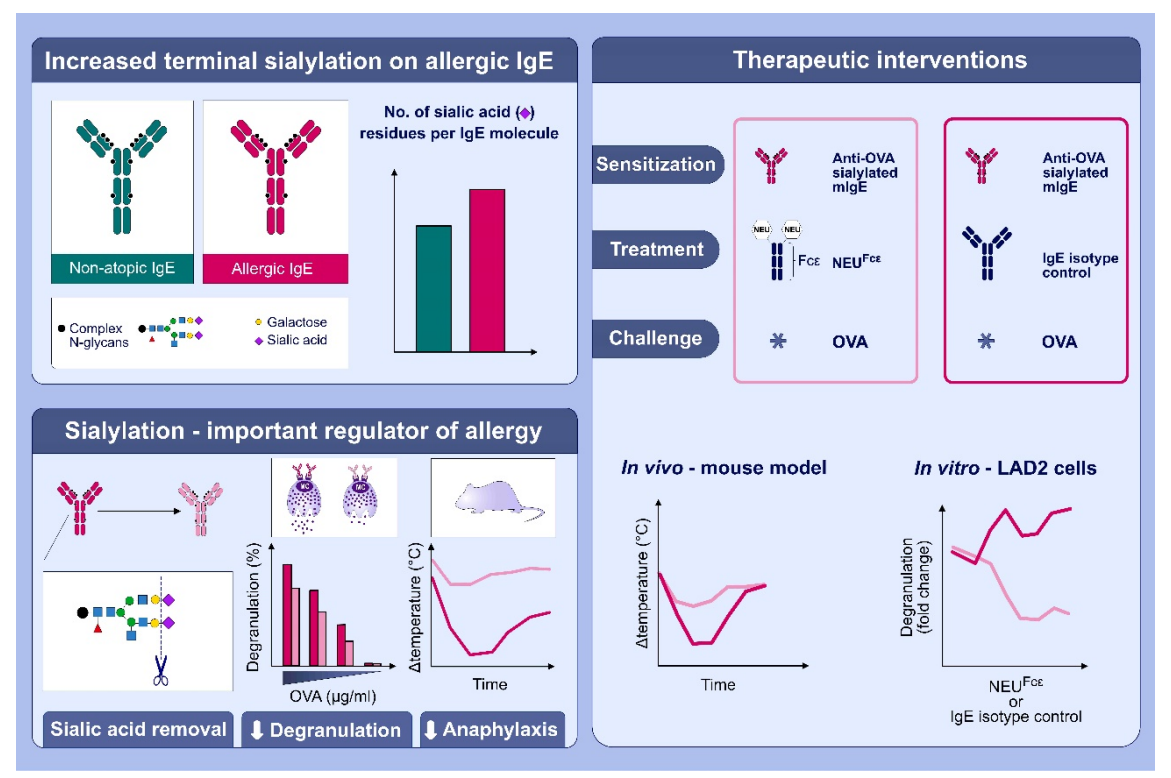

Conflict of interest : The authors declare that they have no conflicts of interest in relation to this manuscript.

Financial support : The authors received no specific funding for this work.

Authorship : All authors contributed to the manuscript, revised and edited.

Acknowledgements : The authors recognize Dr. Anna Globinska for graphical abstract design and Dr. Rodrigo Jiménez-Saiz for critical review of the manuscript.

\section{References :}

1.- Epp A, Sullivan KC, Herr AB, Strait RT. Immunoglobulin Glycosylation Effects in Allergy and Immunity. Curr Allergy Asthma Rep. 2016;16(11):79.

2.- Shade KT, Conroy ME, Anthony RM. IgE Glycosylation in Health and Disease. Curr Top Microbiol Immunol. 2019;423:77-93.

3.- Arnold JN, Radcliffe CM, Wormald MR, et al. The glycosylation of human serum IgD and IgE and the accessibility of identified oligomannose structures for interaction with mannan-binding lectin. J Immunol. 2004;173(11):6831-6840.

4.- Gould HJ, Sutton BJ. IgE in allergy and asthma today. Nat Rev Immunol. 2008;8(3):205-217. 5.- Niki T, Tsutsui S, Hirose S, et al. Galectin-9 is a high affinity IgE-binding lectin with anti-allergic effect by blocking IgE-antigen complex formation. J Biol Chem. 2009;284(47):32344-32352.

6.- Shade KT, Platzer B, Washburn N, et al. A single glycan on IgE is indispensable for initiation of anaphylaxis. J Exp Med. 2015 Apr 6;212(4):457-67.

7.- Shade KC, Conroy ME, Washburn N, et al. Sialylation of immunoglobulin E is a determinant of allergic pathogenicity. Nature. 2020;582(7811):265-270.

8.- Petry J, Rahmöller J, Dühring L, et al. Enriched blood IgG sialylation attenuates IgG-mediated and IgGcontrolled-IgE-mediated allergic reactions. J Allergy Clin Immunol. 2020 Jun 27:S0091-6749(20)30887-3.

9.- Crawford G, Hayes MD, Seoane RC, et al. Epithelial damage and tissue $\gamma \delta \mathrm{T}$ cells promote a unique tumor-protective IgE response. Nature Immunology. 2018 Aug;19(8):859-870. 